\title{
ON LOCAL CONVEXITY IN HILBERT SPACE
}

\section{J. SCHOENBERG}

1. Introduction. Let $M$ be a set of points in a normed vector space $V$. $M$ is said to be convex if $p$ and $q$ being any two points of $M$, the whole rectilinear segment $\overline{p q}$ belongs to $M$. A weakened form of convexity, due to $H$. Tietze, ${ }^{1}$ is as follows:

Definition of LOCAL CONVEXITy. Let $p \in M$. The set $M$ is said to be locally convex at $p$ if there exists a positive $\rho=\rho(p)$ such that the intersection $M \cdot S(p ; \rho)$, of $M$ with the open sphere $S(p ; \rho)$ of center $p$ and radius $\rho$, is convex in the ordinary sense. The set $M$ is called locally convex if $M$ is locally convex at all its points.

Every convex set is locally convex. The converse is not true since every open set is obviously locally convex. Tietze's chief result concerning local convexity is as follows:

Theorem 1 (of Tietze). Let $E_{k}$ denote the k-dimensional euclidean space. A closed and connected set $M$ in $E_{k}$ which is locally convex is also convex in the ordinary sense.

By means of his concept of local euclidean dimension of a set $M$ at a point $p \in M$, Tietze reduces the proof of Theorem 1 to the case of locally convex continua with interior points and which coincide with the closure of their set of interior points. Tietze then proves the theorem for continua in $E_{2}$ and finally extends the proof to cover any $E_{k}$. Tietze's method does not seem to be applicable for sets in Hilbert space.

The following lines contain a simpler method of dealing with this problem which allows the establishment of Tietze's theorem in any real or complex normed vector space whether separable or not. For the sake of definiteness we state and prove our theorem for real Hilbert space. ${ }^{2}$

Presented to the Society, September 8, 1939 under the title On local convexity in euclidean spaces; received by the editors August 13,1941. The present note is an improved version (see Footnote 6).

${ }^{1} \mathrm{H}$. Tietze, Über Konvexheit im kleinen und im grossen und über gewisse den Punkten einer Menge zugeordneten Dimensionszahlen, Mathematische Zeitschrift, vol. 28 (1928), pp. 697-707.

2 Recent results have demonstrated the breakdown of some classical properties of convex sets when we pass from euclidean spaces to Hilbert spaces. See, for example, David Moskowitz and L. L. Dines, Convexity in linear spaces with an inner product, Duke Mathematical Journal, vol. 5 (1939), pp. 520-534, in particular the example on 
TheOREM 2. A closed and connected set $M$ in the real Hilbert space $H$ which is locally convex is also convex in the ordinary sense.

2. On $\delta$-convexity and three lemmas. All our sets are in Hilbert space unless otherwise stated. A strengthened form of local convexity is as follows:

Definition of $\delta$-Convexity. ${ }^{3}$ Let $\delta$ be a positive number. $A$ set is said to be $\delta$-convex if $p$ and $q$ being any two points of $M$, of distance $p q<\delta$, the whole segment $\overline{p q}$ belongs to $M$.

It is clear that $\delta$-convexity implies local convexity. The converse is not true. Thus the following two sequences of points on the $x$-axis (space $E_{1}$ )

$$
\begin{array}{ll}
M_{1}:\left\{\frac{1}{n}\right\}, & n=1,2, \cdots, \\
M_{2}:\left\{1+\frac{1}{2}+\cdots+\frac{1}{n}\right\}, & n=1,2, \cdots,
\end{array}
$$

are clearly locally convex but not $\delta$-convex, no matter how small $\delta$ may be. Notice that $M_{1}$ is bounded but not closed and $M_{2}$ is closed but not bounded, hence none of these two sets is self-compact. For self-compact sets both types of convexity are equivalent, a fact which we now state.

Lemma 1. A self-compact set $M$ which is locally convex is also $\delta$-convex for some appropriate value of $\delta$.

Indeed, the contrary assumption to the effect that $M$ is never $\delta$-convex, no matter how small $\delta$ is, implies that for every integer $n$ our set $M$ contains a pair of points $a_{n}, b_{n}$ with $a_{n} b_{n}<1 / n$ and such that the segment $\overline{a_{n} b_{n}}$ does not wholly belong to $M$. Since $M$ is self-compact we may assume $a_{n} \rightarrow p, b_{n} \rightarrow p$ and $p \in M$. But this clearly contradicts the local convexity of $M$ at the limiting point $p$.

pp. 531-532. This makes it the more remarkable that Tietze's theorem does hold in Hilbert space.

3 The $\delta$-convexity is readily seen to be equivalent to uniform local convexity. By this we mean local convexity of a set in the sense of our first definition with a radius $\rho=\rho(p)>0$ which is independent of $p$.

A $\delta$-convex set is a very special instance of a metric space with elementary arcs in the sense of Marston Morse, The Calculus of Variations in the Large, American Mathematical Society Colloquium Publications, vol. 18, New York, 1934, p. 298, See also J. H. C. Whitehead, Convex regions in the geometry of paths, Quarterly Journal of Mathematics, vol. 3 (1932), pp. 33-42. 
Our principal tool will be the following elementary geometrical lemma in euclidean space:

Lemma $2 .^{4}$ Let $p_{0}, p_{1}, \cdots, p_{n}\left(p_{0} \neq p_{n}\right)$ be points in the euclidean space $E_{k}$. Let II denote the open polygonal line joining successively the given points $p_{0}, p_{1}, \cdots, p_{n}$. Regarding $E_{k}$ as a vector space we derive a new sequence of points by setting

$$
\begin{array}{rlrl}
p_{-1}^{\prime}=p_{0}, & p_{0}^{\prime}=\frac{1}{2}\left(p_{0}+p_{1}\right), & p_{1}^{\prime}=\frac{1}{2}\left(p_{1}+p_{2}\right), \cdots, \\
p_{n-1}^{\prime}=\frac{1}{2}\left(p_{n-1}+p_{n}\right), & p_{n}^{\prime}=p_{n} .
\end{array}
$$

Repeating the same operation on the newly derived broken line $\Pi^{\prime}$ $=p_{-1}^{\prime} p_{0}^{\prime} \cdots p_{n}^{\prime}$, let

$$
\begin{gathered}
p_{-2}^{\prime \prime}=p_{-1}^{\prime}=p_{0}, \quad p_{-1}^{\prime \prime}=\frac{1}{2}\left(p_{-1}^{\prime}+p_{0}^{\prime}\right), \quad p_{0}^{\prime \prime}=\frac{1}{2}\left(p_{0}^{\prime}+p_{1}^{\prime}\right), \cdots, \\
p_{n-1}^{\prime \prime}=\frac{1}{2}\left(p_{n-1}^{\prime}+p_{n}^{\prime}\right), \quad p_{n}^{\prime \prime}=p_{n}^{\prime}=p_{n} .
\end{gathered}
$$

Repeating this operation indefinitely we obtain an infinite sequence of broken lines

$$
\Pi^{(m)}=p_{-m}^{(m)} p_{-m+1}^{(m)} \cdots p_{n}^{(m)}, \quad m=1,2,3, \cdots,
$$

all joining the points $p_{0}$ and $p_{n}$. Let $N$ be an open set containing the closed segment $\overline{p_{0} p_{n}}$. Then $N$ will contain the whole broken line $\Pi^{(m)}$ for all sufficiently large values of $m$.

Since our proof will be independent of the dimension $k$ we may without loss of generality assume that $k=2$. Let $p_{i}^{(0)}=p_{i}$ and $\Pi^{(0)}=\Pi$. For convenience we now extend the definition of the points $p_{i}^{(m)}$ $(-m \leqq i \leqq n)$ for all integral values of $i(=0, \pm 1, \pm 2, \cdots)$ by setting

$$
\begin{aligned}
& p_{i}^{(m)}=p_{-m}^{(m)}=p_{0} \quad \text { if } \quad i \leqq-m, \\
& p_{i}^{(m)}=p_{n}^{(m)}=p_{n} \quad \text { if } \quad i \geqq n .
\end{aligned}
$$

We then have without exception the relation

$p_{i}^{(m)}=\frac{1}{2}\left(p_{i}^{(m-1)}+p_{i+1}^{(m-1)}\right), \quad m=1,2, \cdots ; i=0, \pm 1, \pm 2, \cdots$, and therefore

$$
p_{i}^{(m)}=\frac{1}{2^{m}}\left\{p_{i}+C_{m, 1} p_{i+1}+C_{m, 2} p_{i+2}+\cdots+p_{i+m}\right\} .
$$

${ }^{4}$ Lemma 2 was suggested by the related problem (Number 3547 ) proposed by Martin Rosenman, American Mathematical Monthly, vol. 39 (1932), p. 239, and by its elegant solution due to R. E. Huston, ibid., vol. 40 (1933), pp. 184-185. 
We now choose in our plane $E_{2}$ a coordinate system $(x, y)$ such that $p_{0}=(0,0), p_{n}=(1,0)$. Furthermore, let the open set $N$ contain the open rectangle

$$
R:-\epsilon<x<1+\epsilon, \quad-\epsilon<y<\epsilon .
$$

Finally let $p_{i}=\left(x_{i}, y_{i}\right)$. In view of (2) and (3) it suffices to establish the two inequalities

$$
\begin{aligned}
& -\epsilon<\frac{1}{2^{m}}\left\{x_{i}+C_{m, 1} x_{i+1}+\cdots+x_{i+m}\right\}<1+\epsilon, \\
& -\epsilon<\frac{1}{2^{m}}\left\{y_{i}+C_{m, 1} y_{i+1}+\cdots+y_{i+m}\right\}<\epsilon,
\end{aligned}
$$

for sufficiently large values of $m$. From (1), for $m=0$, we get

$$
\begin{array}{ll}
\cdots=x_{-2}=x_{-1}=x_{0}=0, & x_{n}=x_{n+1}=x_{n+2}=\cdots=1, \\
\cdots=y_{-2}=y_{-1}=y_{0}=0, & y_{n}=y_{n+1}=y_{n+2}=\cdots=0 .
\end{array}
$$

But then our inequalities (4) and (5) obviously hold for sufficiently large values of $m$, for the sum of any set of at most $n-1$ consecutive ones among the binomial coefficients $1, C_{m, 1}, C_{m, 2}, \cdots, 1$, divided by their total sum $2^{m}=1+C_{m, 1}+\cdots+1$, tends uniformly to zero as $m \rightarrow \infty$. Hence our lemma is proved. ${ }^{5}$

Our geometrical Lemma 2 will be made effective for our purpose by the following third and last lemma which states the effect of the assumption of local convexity on connected sets.

Lemma 3. Let $M$ be a set in Hilbert space which is closed, connected and locally convex. Then $M$ is also connected in the following stronger sense: Any two points $a$ and $b$ of $M$ may be joined by a finite polygonal line lying wholly in $M .^{6}$

Indeed, denote by $N$ the set of points of $M$ which may be joined to the fixed point $a$ by a finite polygonal line lying in $M$.

${ }^{5} \mathrm{~A}$ proof of Theorem 2 in the complex Hilbert space requires a proof of Lemma 2 in the $k$-dimensional complex euclidean (unitary) space $U_{k}$. The proof just given is readily adaptable to the space $U_{2}$ of two complex coordinates $x=x^{\prime}+i x^{\prime \prime}, y=y^{\prime}+i y^{\prime \prime}$. The only change is that the rectangular neighborhood (3) of the segment $\overline{p_{0} p_{n}}$ is to be replaced by the open set defined by $-\epsilon<x^{\prime}<1+\epsilon,-\epsilon<x^{\prime \prime}<\epsilon,-\epsilon<y^{\prime}<\epsilon$, $-\epsilon<y^{\prime \prime}<\epsilon$. This is essentially again the real case for the space $E_{4}$.

${ }^{6}$ Open connected sets are known to enjoy this stronger connectivity property. In fact Lemma 3 implies this classical property since open sets are locally convex as already mentioned.

Lemma 3 was pointed out to the author by S. Kakutani and J. W. Tukey. The earlier version of this paper was thereby simplified and the method became applicable to Hilbert space. 
$N$ is closed in $M$. Indeed, let $q_{i} \rightarrow q, q_{i} \in N, q \in M$. Since $M \cdot S(q ; r)$ is convex for a certain $r>0$, as soon as $q_{i} \in S(q ; r)$ we have $\overline{q_{i} q} \subset M$, hence $q \in N$.

$N$ is open in $M$. For if $q \in N$ clearly all points of $M \cdot S(q ; r)$ will be in $N$, provided $r$ is sufficiently small.

The connectedness of $M$ now implies that $N=M$.

3. Proof of Theorem 2. Let $M$ be a closed and connected set in $H$ which is also locally convex. Let $a$ and $b$ be any two points of $M$. We want to show that $\overline{a b} \subset M$. By Lemma 3 there is a sequence of points $p_{1}, \cdots, p_{n-1}$ such that the polygonal line $\Pi=a p_{1} p_{2} \cdots p_{n-1} b$ lies in $M$. Let $E_{n}$ be a euclidean subspace of $H$ which contains this polygonal line $\Pi$. Let furthermore $\bar{S}(a ; r)$ be a closed sphere of $H$ of sufficiently large radius $r$ so as to contain our polygonal line II. The intersection

$$
M_{1}=M \cdot E_{n} \cdot \bar{S}(a ; r)
$$

is obviously closed and bounded, hence also self-compact as being a subset of $E_{n}$. But $M_{1}$ is also locally convex, being the intersection of three locally convex sets. By Lemma 1 we know that $M_{1}$ is $\delta$-convex for some appropriate value of $\delta$. On the other hand our assumptions insure that $\Pi \subset M_{1}$. By subdividing, if necessary, the sides of $\Pi$ we may assume that the sides $p_{i} p_{i+1}$ of the line $\Pi$ are all of length less than $\delta$. We now perform the construction of Lemma 2. Since $\Pi \subset M_{1}$, the $\delta$-convexity of $M_{1}$ and the triangle inequality imply that also $\Pi^{\prime} \subset M_{1}$. Since all sides of all derived polygonal lines $\Pi^{(m)}$ are of length less than $\delta$, induction with respect to $m$ will show that $\Pi^{(m)} \subset M_{1}$ for all values of $m$. By Lemma $2 \Pi^{(m)}$ converges to the segment $\overline{a b}$ as $m \rightarrow \infty$. Since $M_{1}$ is closed we indeed have $a b \subset M_{1}$, hence $\overline{a b} \subset M$. Thus $M$ is convex and our theorem is proved.

University of Pennsylvania 